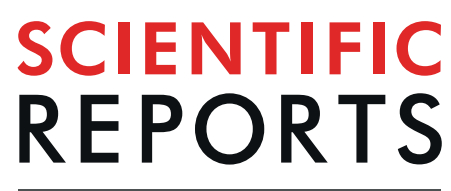

natureresearch

Check for updates

\title{
Focussing Protons from a Kilojoule Laser for Intense Beam Heating using Proximal Target Structures
}

\author{
C. McGuffey ${ }^{1} \bowtie$, J. Kim ${ }^{1}$, M. S. Wei ${ }^{2}$, P. M. Nilson ${ }^{3}$, S. N. Chen 4,6 , J. Fuchs ${ }^{4}$, P. Fitzsimmons ${ }^{2}$, \\ M. E. Foord ${ }^{5}$, D. Mariscal ${ }^{1,5}$, H. S. McLean ${ }^{5}$, P. K. Patel ${ }^{5}$, R. B. Stephens ${ }^{2}$ \& F. N. Beg ${ }^{1}$
}

Proton beams driven by chirped pulse amplified lasers have multi-picosecond duration and can isochorically and volumetrically heat material samples, potentially providing an approach for creating samples of warm dense matter with conditions not present on Earth. Envisioned on a larger scale, they could heat fusion fuel to achieve ignition. We have shown in an experiment that a kilojoule-class, multipicosecond short pulse laser is particularly effective for heating materials. The proton beam can be focussed via target design to achieve exceptionally high flux, important for the applications mentioned. The laser irradiated spherically curved diamond-like-carbon targets with intensity $4 \times 10^{18} \mathrm{~W} / \mathrm{cm}^{2}$, producing proton beams with $3 \mathrm{MeV}$ slope temperature. A Cu witness foil was positioned behind the curved target, and the gap between was either empty or spanned with a structure. With a structured target, the total emission of $\mathrm{Cu} \mathrm{K} \alpha$ fluorescence was increased 18 fold and the emission profile was consistent with a tightly focussed beam. Transverse proton radiography probed the target with ps order temporal and $10 \mu m$ spatial resolution, revealing the fast-acting focussing electric field. Complementary particle-in-cell simulations show how the structures funnel protons to the tight focus. The beam of protons and neutralizing electrons induce the bright $\mathrm{K} \alpha$ emission observed and heat the $\mathrm{Cu}$ to $100 \mathrm{eV}$.

High-intensity proton beams generated by ultrashort pulse laser-matter interactions ${ }^{1-3}$ were immediately recognized as a powerful tool for the creation of Warm Dense Matter (WDM ${ }^{4,5}$. These beams have since found widespread use in High Energy-Density physics studies as isochoric heaters that allow study of conditions similar to those in the interior of planets ${ }^{6}$, as probes of complex objects ${ }^{7}$ and of transient electric and magnetic fields ${ }^{8-11}$ with micron scale resolution ${ }^{12}$, or for inducing nuclear reactions to create directional neutron beams ${ }^{13,14}$. These intense proton beams also hold promise as the ignitor in Fast Ignition (FI) fusion ${ }^{15-17}$ if the total beam energy can be scaled up.

The utility of these beams comes from their high particle energy (10's of MeV) and energy bandwidth, low source emittance ${ }^{18}$, and short (multi-ps) initial pulse duration produced by the target normal sheath acceleration (TNSA) mechanism ${ }^{3}$. The beams initially have a pulse duration similar to the laser duration down to a few picoseconds. A bunch length can then be estimated for different source-to-sample distances by considering the velocity dispersion of the broadband beam. While advanced acceleration mechanisms are predicted to produce very high energy protons and ions ${ }^{19-22}$, the well-established TNSA scheme has a distinct advantage in that the beams are very easy to produce and simply curving the target can focus the beam. TNSA-hybrid mechanisms hold the current record for highest energy, up to $100 \mathrm{MeV}^{23,24}$.

Focussing the ion beam increases the flux and widens the possibilities for all the above-listed applications. The extremely low beam transverse emittance of TNSA allows a curved hemisphere target to focus the proton beam to small spot size $(50-100 \mu \mathrm{m})$, as demonstrated on small $\left(10 \mathrm{~J}, 100 \mathrm{ss}^{4}, 75 \mathrm{~J}, 700 \mathrm{~s}^{25}\right)$ and medium scale facilities $\left(400 \mathrm{~J}, 400 \mathrm{fs}^{26}, 170 \mathrm{~J}, 700 \mathrm{fs}^{5}\right)$. However, the ultimate achievable focussing is limited by hot electron pressure in the radially shrinking proton beam itself ${ }^{27}$. The consequence of this is that for high charge, tightly focussed beams,

${ }^{1}$ Center for Energy Research, University of California, San Diego, La Jolla, CA, 92093-0417, USA. ${ }^{2}$ General Atomics, P.O. Box 85608, San Diego, CA, 92186-5608, USA. 'Laboratory for Laser Energetics, University of Rochester, Rochester, NY, 14623, USA. 'Laboratoire pour I'Utilisation des Lasers Intenses - CNRS, CEA, UPMC Univ Paris 06: Sorbonne Université, Ecole Polytechnique, Institut Polytechnique de Paris, F-91128, Palaiseau cedex, France. ${ }^{5}$ Lawrence Livermore National Laboratory, P.O. Box 808, Livermore, CA, 94550, USA. ${ }^{6}$ Extreme Light Infrastructure - Nuclear Physics/Horia Hulubei National Institute for R\&D in Physics and Nuclear Engineering, Bucharest-Magurele, 077125, Romania. ${ }^{\varpi}$ e-mail: cmcguffey@ucsd.edu 


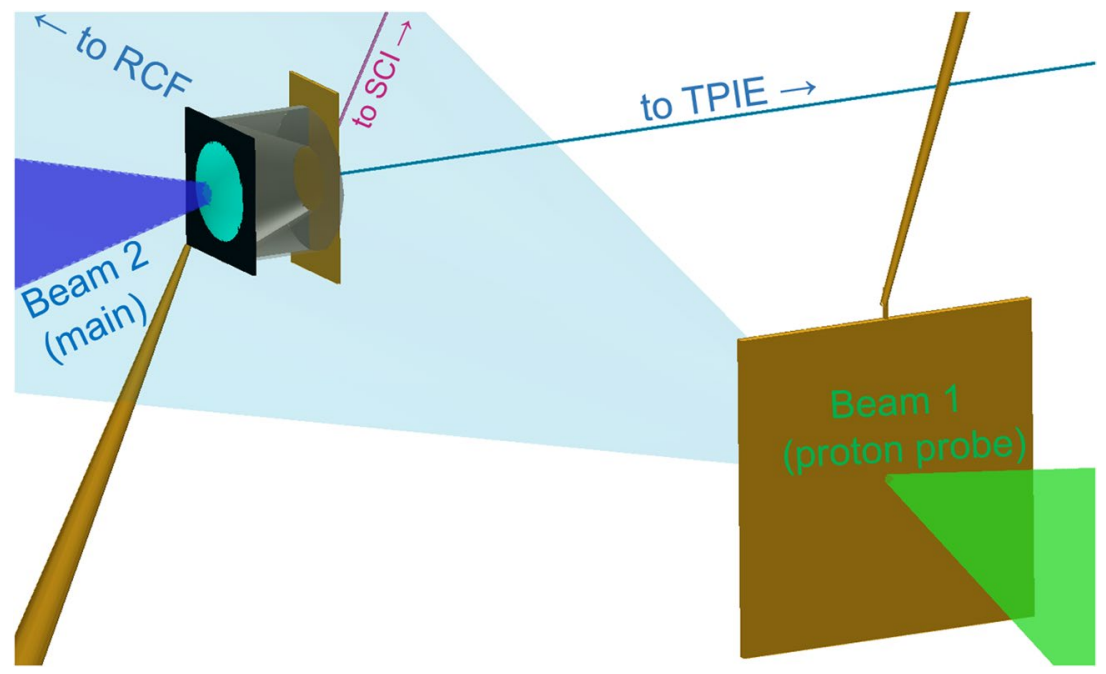

Figure 1. Experimental configuration drawn with VISRAD software. OMEGA EP short pulse main beam irradiated a curved diamond-like carbon target attached to an Al cone to produce an ultrahigh intensity proton beam, directed into various transport media. The cone is shown cutaway. A Cu diagnostic layer was glued to the back of the target. The other short pulse beam produced a transverse proton probing beam shown as a transparent cone pointing toward the RCF stack. Lines of sight toward TPIE and the SCI x-ray imager as shown as thin lines. Stalks supporting each target are shown as brown, tapered lines. For the freestanding case, a third stalk supported the $\mathrm{Cu}$ foil.

the protons do not move ballistically through a focus, and a spherically curved target is not enough, by itself, to achieve optimal focussing ${ }^{28}$. Early experimental data on the kiloJoule OMEGA EP laser showed that proton beam focussing from free-standing curved targets was indeed limited by this non-ballistic behavior at this higher energy laser drive ${ }^{29}$.

However, the paper by Bartal et al. also discovered that by attaching a cylindrical or conical structure to the target, the beam could again be tightly focussed. Simulations showed that this was caused by a focussing electrostatic field that rapidly-grew on the inside of the structure as hot electrons from the interaction escaped the target and populated a sheath along the structure walls $\mathrm{s}^{30,31}$. Related field effects have been studied in detail for periodic target structures with a $\tau=30 \mathrm{fs}$ laser ${ }^{32}$. The finding that the cone focussed the beam was particularly appealing for FI, which is often envisioned with a cone embedded in a spherical target. It is also broadly appealing for high beam current applications to overcome the beam pressure limitation because focussing caused by the cone can be expected to be more significant as the laser energy and pulse duration are increased, motivating the experiment presented here.

In this work, we show a highly effective method for focussing protons and heating a secondary target using conical-shaped target structures with fundamentally different laser conditions than previous proton heating experiments. The high energy and pulse duration of the OMEGA EP drive laser produced a proton beam that, when focussed, achieved exceptionally high peak beam density. This dramatically increases the prospects for using protons for isochoric heating and the intensity-hungry applications mentioned above. This is an important demonstration that a structure behind the target is still effective for focussing a proton beam from a much higher energy and longer duration laser than the Bartal result. Furthermore, the OMEGA EP orthogonal short pulse beam was used to drive a second beam of protons which radiographed the fields responsible for focussing. In the second half of the paper, we present particle in cell simulations that confirm the focussing and show the mechanism in detail. In two-step simulations, the heating of a secondary target is evaluated, with an expected temperature of $100 \mathrm{eV}$. To conclude, we show how a steeper cone geometry may increase heating and constrict the proton beam to the size required for proton FI.

\section{Findings}

Proton focussing measurements were taken for the first time in the kiloJoule regime at the OMEGA EP facility using a $1.25 \mathrm{~kJ}, 10$ ps short pulse laser along with proton spectrum measurements using a Thomson parabola diagnostic named Thomson Parabola Ion Energy Analyzer (TPIE). The laser was incident on a diamond-like carbon spherical cap ("hemi"). Protons were focussed into metallic foils and their transmitted spectrum was recorded. The flux was sufficient to induce bright $\mathrm{K} \alpha \mathrm{x}$-rays from the rear $\mathrm{Cu}$ layer as measured by a single-hit spectrometer and a Spherical Crystal Imager (SCI). A second, orthogonal OMEGA EP beam irradiated flat foils with $850 \mathrm{~J}$ in $10 \mathrm{ps}$ in order to probe the interaction with broad-energy protons of order $\mathrm{MeV}$. Due to the protons' velocity dispersion, different energy protons probed the interaction plane at different delays. They were then discriminated by energy using a stack of RadioChromic Film (RCF) detectors. The different layers of film, or 'frames', resolve the target dynamics as the laser arrives with resolution of a few ps. Further details about the laser, target, and diagnostics are given in the Methods Section while the experimental configuration is illustrated in Fig. 1. 


\section{free wedge cone}
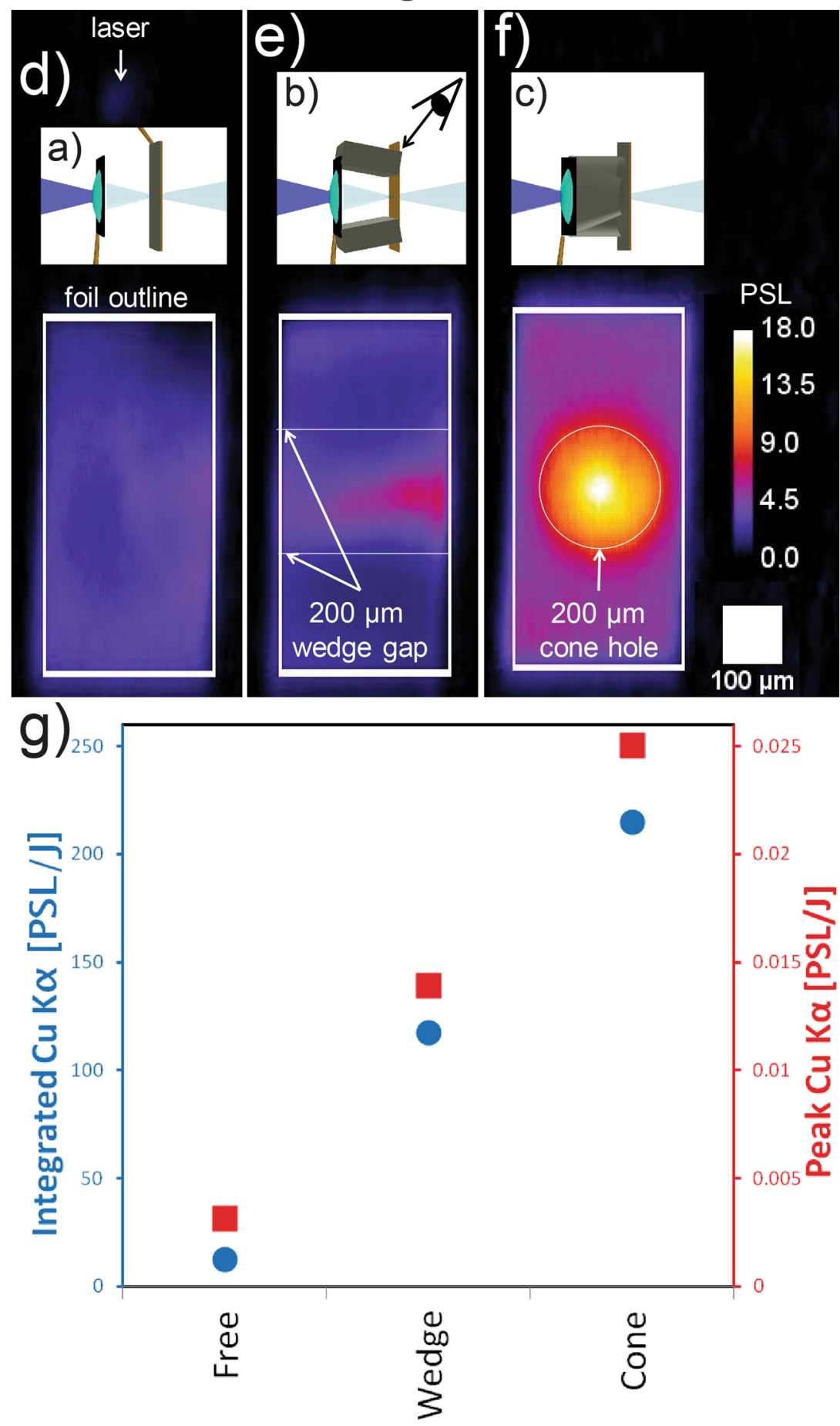

Figure 2. Three target types (insets (a-c) drawn with VISRAD software) used in the OMEGA EP experiment, and their corresponding emission profiles of $8.048 \mathrm{KeV} \mathrm{Cu} \mathrm{K \alpha}(\mathbf{d}-\mathbf{f})$. (g) Plot of the emission brightness from the foil center (peak pixel) and integrated over the full foil dimension. The unit PSL stands for Photo-Stimulated Luminescence, the standard measure of the deposited energy in the imaging plate detector. The SCI viewing position for all cases is illustrated in (b). For orientation, note the dim signal in (d) (above inset (a)) which is due to hemi self-emission and indicates the laser interaction point.

The SCI data are shown in Fig. 2 for the three target types. For the case of freestanding, separated foils, illustrated in Fig. 2(a), the $\mathrm{Cu} \mathrm{K} \alpha$ signal was weak and diffuse over the entire $\mathrm{Cu}$ foil, Fig. 2(d). This confirmed that the inherently diverging electron beam from the interaction contributed minimally to the $\mathrm{Cu} \mathrm{K} \alpha$ signal at this 


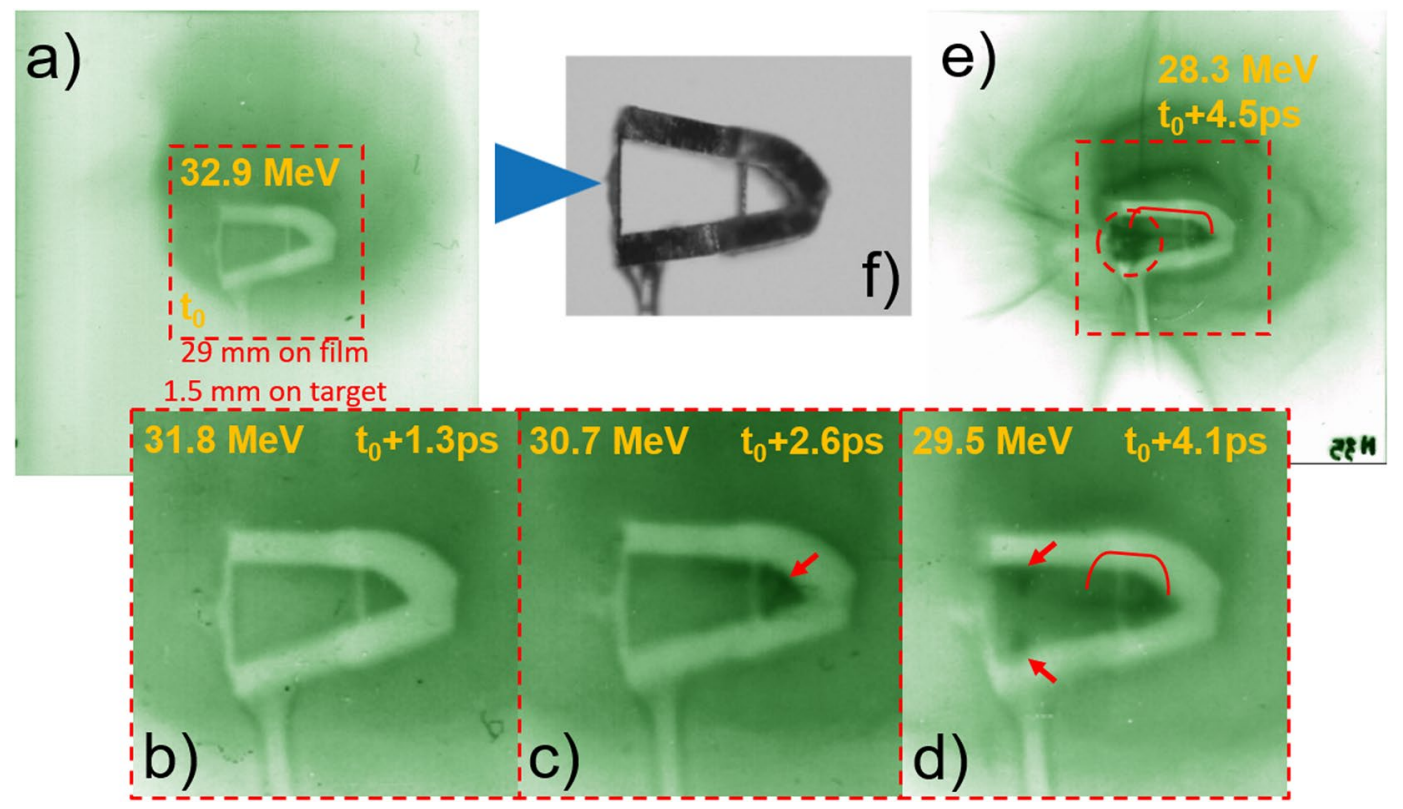

Figure 3. Proton radiographs showing picosecond-timescale dynamics as the main target is irradiated. (f) shows a photograph of a folded wedge target. A sequence of five images from the same shot are shown with relative times as indicated $(\mathbf{a}-\mathbf{e})$. Three consecutive frames $(\mathbf{b}-\mathbf{d})$ are zoomed to show excess dose (darkening) around the rear inner walls (arrows) and a band on the center plane (bracket) indicating a focussing field between the top and bottom wedge segments. By 4.5 ps into the interaction (e), the probe protons accumulate into a dark patch (circle). Meanwhile, the target becomes rapidly charged, driving a current in the stalk and launching expanding sheaths.

standoff distance. Signal was darker in the top-right corner, corresponding to the stalk connection point, visible in Fig. 2(a). In contrast to this diffuse signal, for the case in which the gap was bridged by a wedged structure, Fig. 2(b), signal was increased on the wedge center plane by $5 \times$ as seen in Fig. 2(e). The emission is greatest in the center, not at the edges of the wedge connection vertices, and no signal enhancement is observed in the region directly in contact with the wedges. This suggests that the signal is due to freestreaming particles within the vacuum gap rather than particles transporting through the wedge. For the case with a cone spanning the gap (c), the effect was further enhanced, Fig. 2(f), with $10 \times$ higher peak signal and $18 \times$ higher integrated signal compared to the freestanding case, Fig. $2(\mathrm{~g})$. These data indicate confinement of the proton beam in one dimension by the wedge and in two dimensions by the cone.

The proton beam spectrum from all targets was collected with the TPIE which was apertured with a pinhole in the forward direction with solid angle $\sim 10^{-7} \mathrm{sr}$. Extrapolation of the full proton beam was made by multiplying the measured solid angle on layers of RCF in a separate shot day. The beam angular distribution was similar shot-to-shot. For a shot with a free hemi with no structure and no foil, the spectrum had a characteristic slope temperature of $T_{p}=2.7 \mathrm{MeV}$, maximum energy $E_{\text {max }}=19 \mathrm{MeV}$ and inferred beam energy $\varepsilon=45 \mathrm{~J}$, or $3.7 \%$ of the laser energy for protons with $E>3 \mathrm{MeV}$. The maximum energy and inferred beam energy drop somewhat for the free hemi case with a metal foil $\left(E_{\max }=16 \mathrm{MeV}, \varepsilon=39 \mathrm{~J}\right)$ and moreso when the wedge and cone structures are attached (with the lowest case being $E_{\max }=12 \mathrm{MeV}, \varepsilon=32 \mathrm{~J}$ ). Example spectra are shown in the Supplementary Information Supp. Fig. 1. The TPIE data also showed a beam of $\mathrm{C}^{6+}$ with nominally $15 \mathrm{x}$ lower signal (PSL) peaking at $15 \mathrm{MeV}$ energy. Such carbon ions would couple effectively to the Cu foil. However, their contribution to heating would be less than from protons due to reduced numbers. Further, the heating would occur after the most intense proton isochoric heating (the fastest $\mathrm{C}$ ion arrives after $25 \mathrm{ps}$ ). Their contribution to $\mathrm{Cu} \mathrm{K} \alpha$ through $\mathrm{C}$ particle-induced X-ray emission (PIXE) is expected to be significantly less than from proton PIXE because the carbons have fewer particles and lower PIXE cross-section based on limited measurements in the literature at lower projectile energy in $\mathrm{Cu}^{33}$ and comparable projectile energy in $\mathrm{Zn}^{34}$.

We can estimate proton beam parameters at the $\mathrm{Cu}$ foil as follows. $32 \mathrm{~J}$ of protons passing through the full aperture of the cone, $200 \mu \mathrm{m}$, during a $20 \mathrm{ps}$ window (i.e. within the time-of-flight from hemi to cone tip for all protons with $>1.5 \mathrm{MeV})$ corresponds to $2 \times 10^{28} \mathrm{p} /\left(\mathrm{cm}^{2} \mathrm{~s}\right)$ luminosity, $1.6 \mathrm{TW}$ power, and $5 \times 10^{15} \mathrm{~W} / \mathrm{cm}^{2}$ intensity. These instantaneous values are comparable to or exceed typical peak values in conventional proton beam sources. For example, a single bunch from the Linac2 injector used at the Large Hadron Collider has peak power $1 G W$. The average power of the laser-driven source is much lower than conventional ion beamlines.

Proton radiography of the wedge case supports the focussing explanation. Five frames are shown in Fig. 3 including three consecutive, zoomed frames. The most interesting feature of the frames is a dark band that originates near the rear of the wedge on the center plane. It develops from the rear and then zips toward the curved target as seen in the sequence. This is explained by a transverse field along the wedge inner walls, directed toward the center plane. The field causes the probe protons above and below the plane to overlap on the detector forming 

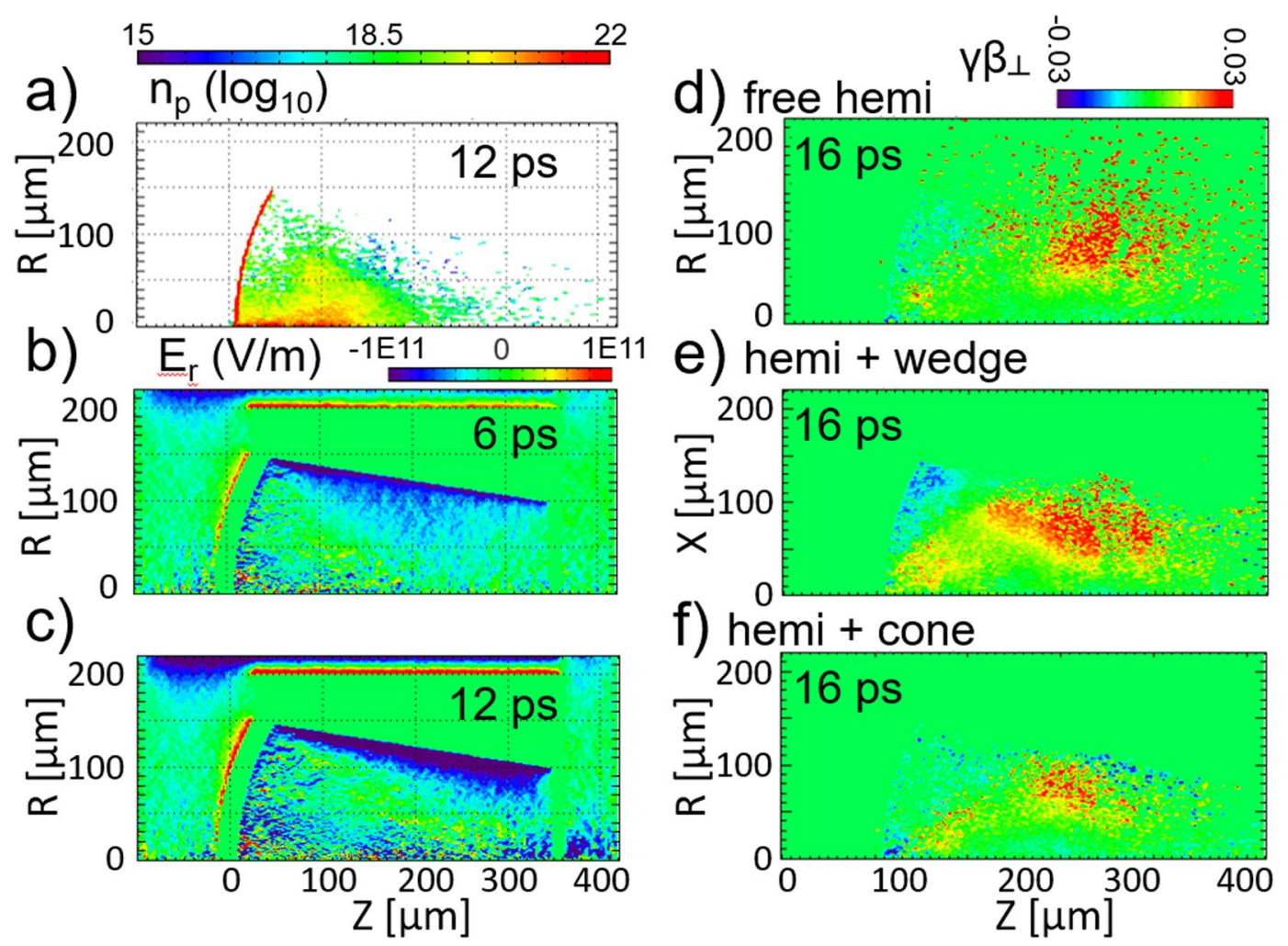

e) hemi + wedge

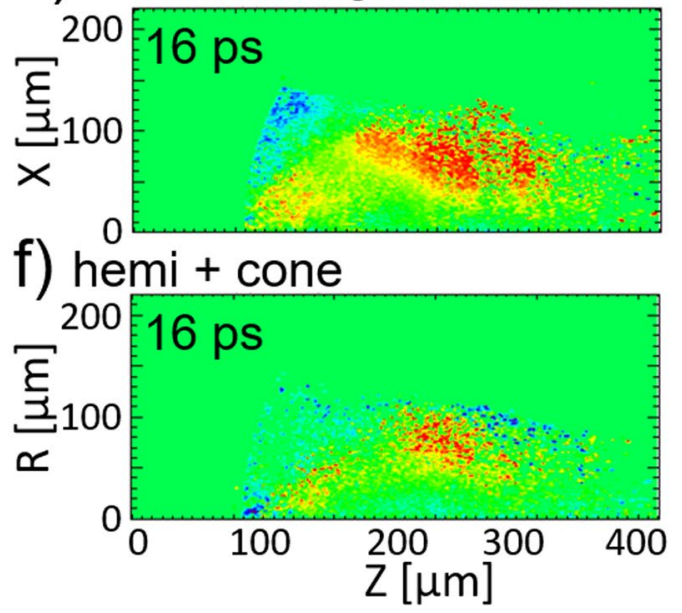

Figure 4. LSP simulations of proton expansion from a hemi and focussing with a rear cone structure using a source of fast electrons to represent the interaction of the OMEGA EP laser. For a hemi + cone case, (a) shows the proton density at time $12 p s ;(\mathbf{b}, \mathbf{c})$ show the transverse electric field $E_{r}$ at $6 p s$ and $12 p s$, respectively. (d-f) Show the macroparticle transverse velocities for the free hemi, hemi + wedge, and hemi + cone cases, respectively.

the dark band (higher exposure) and they zip together from the back to front because the top and bottom fields are close together at the rear. This is the same field that focusses the protons from the hemi in the wedge and cone cases. Additionally, a dark patch was observed in the space behind the hemi and between the wedges. These features persist in the subsequent 21 frames $\left(\right.$ til $\left.t_{0}+64 \mathrm{ps}\right)$ after which the films are saturated. Deflection of probe protons was evaluated using a particle in cell pusher with prescribed fields described in the Supplementary Information.

A two step process was applied to simulate the experiment using the particle in cell code LSP in two dimensions (2D) $)^{35}$. The first step studied the accelerating and focussing dynamics. Three cases are presented: the cone freestanding target case simulated and the conical target case using $2 \mathrm{D}$ cylindrical coordinate systems, and the wedge case in a 2D cartesian system. The configuration details are given in the Methods Section. In the freestanding case, protons are initially focussed due to the curvature of the target. However, a significant fraction of them diverge away Fig. 4(d). In the wedge and cone cases, a strong focussing field is observed to persist along the inner cone surface. For the cone case the radial focussing field strength (seen during and after the laser in Fig. 4(b,c) is comparable to the initial field from the curved target, confining protons Fig. 4(b), near the hemi/cone conjunction). Figure 4(d-f) show macroparticle velocities 16 ps into the simulations for the three cases. For the free hemi case, it is clear that the beam is already diverging at $Z=200 \mu \mathrm{m}$, even before the nominal focal position for the curved target, and many particles are more lateral than the radius of the hemi. For the wedge case, the walls of the wedge are a physical barrier to protons. For the cone case, particles can be seen to reflect toward the central axis, corralling and collimating the proton beam.

We can again estimate beam parameters by checking the numbers in the simulations. By integrating the profile of the beam at the back of the cone, we find that $5 \times 10^{13}$ protons passed through a disk-shaped plane of $100 \mu \mathrm{m}$ diameter in $41 \mathrm{ps}$ with an average energy of $1.2 \mathrm{MeV}$. Thus, the beam density averaged across the cone hole and averaged during this time is $5 \times 10^{27} \mathrm{p} /\left(\mathrm{cm}^{2} s\right)$ and the intensity is $7 \times 10^{14} \mathrm{~W} / \mathrm{cm}^{2}$. During a $20 \mathrm{ps}$ window $(96 \%$ of the protons reaching the extraction plane arrived during 21-41 ps) and looking inside $30 \mu \mathrm{m}$ radius, the beam density is $3 \times 10^{28} \mathrm{p} /\left(\mathrm{cm}^{2} \mathrm{~s}\right)$. The highest intensity is $1 \times 10^{16} \mathrm{~W} / \mathrm{cm}^{2}$. These values are in near agreement to the estimates based on the TPIE detailed above. We note that at the end of the 41 ps simulation many slow particles that carry significant energy but that do not contribute to the most intense phase of the interaction have not reached the extraction plane.

In the second modeling step, deposition and induced $K \alpha$ photons in the rear Cu layer are studied. In each of the three cases above, the electrons and protons that made it to the rear foil were recorded and injected into a 


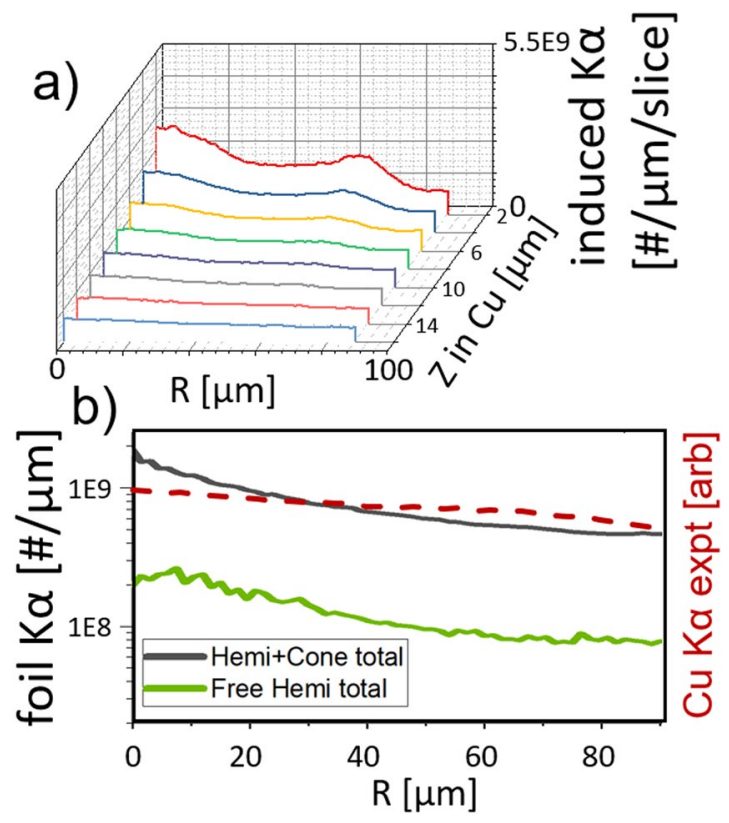

Figure 5. $\mathrm{K} \alpha$ post-processing in the $\mathrm{Cu}$ transport simulation. (a) lateral profiles of proton-induced $\mathrm{Cu} K \alpha$ photons at $2 \mu \mathrm{m}$ intervals in a hemi with wedge case. (b) composite lateral profile for a $10 \mu \mathrm{m}$ foil for the free hemi case and hemi with cone case. An experimental lineout of the hemi-cone case is overlaid.

$\mathrm{Cu}$ slab (see Methods for details). The time-integrated $\mathrm{Cu} \mathrm{K} \alpha$ binned by lateral position and depth into the foil is shown in Fig. 5(a). The photons can be traced back to three distinct populations of particles. Hot electrons, protons, and quasi-neutralizing electrons that co-move with the protons. Hot electrons moving through the cone contribute a non-negligible but uniform $\mathrm{K} \alpha$ signal while the latter two produce a centrally peaked $\mathrm{Cu} \mathrm{K} \alpha$ signal. Figure 5(b) shows that the hemi with cone results in $10 \times$ higher peak $\mathrm{Cu} \mathrm{K} \alpha$ signal than the free hemi case, in accordance with the experimental measurement.

As an additional test of the focussing possibilities, a radial simulation was carried out with a steeper cone angle of $20^{\circ}$ as portrayed in Fig. 6(a). Comparing Fig. 6(b,c), it can be seen that the proton beam is significantly stronger on axis for the steep cone case than the free hemi. Figure $6(\mathrm{~d})$ shows that the majority of proton macroparticles are reflected toward the axis near the cone tip as compared to the other cases in Fig. 4(d-f). Figure 6(e) shows that the standard cone focusses $5 \times$ the number of particles to the axis as the free hemi, while the steep cone would produce even tighter focussing. In the $\mathrm{Cu}$ transport simulations it can be seen how the proximal target structures enhance heating of a sample. Figure 6(f) shows the radial temperature profile in the Cu mid-plane for the three cases. The hemi with standard cone heats to a 15-20\% higher temperature than the free hemi would for radii out to $100 \mu \mathrm{m}$, while the $20^{\circ}$ cone heats to at least twice the nominal temperature for radii out to $40 \mu \mathrm{m}$. The temperature difference between the free hemi and standard cone case at this position is not as striking as the particle distribution 6(e) nor the $\mathrm{Cu} K \alpha$ data because the latter two are weighted to the numerous protons entering the $\mathrm{Cu}$ with $10-1000 \mathrm{keV}$ that do not penetrate to the $\mathrm{Cu}$ mid-plane.

\section{Conclusion}

We have shown that the proton beam from a $1.25 \mathrm{~kJ}, \tau=10 \mathrm{ps}$ laser could be focussed effectively using a conical structure. Direct evidence of focussing is seen in imaged $K \alpha$ emission from a $\mathrm{Cu}$ foil placed at the end of the cone. For the case with a structured target the total emission of $\mathrm{Cu} \mathrm{K} \alpha$ fluorescence was increased by a factor of 18 in experiment. Simulations show that electrostatic, persistent fields on the cone inner wall funnel protons to the cone tip and that the $K \alpha$ signal increase is a combination of extra signal due to electrons directly moving through the target but also a significant constriction of protons into a collimated spot of roughly the cone tip size. We note that the simulation provided valuable identification of the beam behaviors, but there may be quantitative disagreements due to the $2 \mathrm{D}$ description especially for the hemi-wedge case. The $10 p s$ pulse duration is critical to the focussing effect since the beam of protons takes $10 \mathrm{~s}$ of $\mathrm{ps}$ to transit the focussing structure. We presented important proton acceleration data in a laser parameter range that has barely been explored and is much more relevant to proton FI than the majority of laser-driven proton studies using freestanding foil targets and sub-picosecond lasers. Based on the measurements the proton intensity is $>1 \%$ of the focussed laser intensity, an important figure of merit for proton FI, and the beam current density at focus is likely high enough to induce modified stopping behaviors predicted by modeling ${ }^{36}$. The simulated steeper cone produced further focussing and heated the Cu foil to $>150 \mathrm{eV}$ in the central $40 \mu \mathrm{m}$ diameter. Further, we hypothesize that a thin-walled cone would be an even more effective lens and retain the benefits of a reduced mass target. 

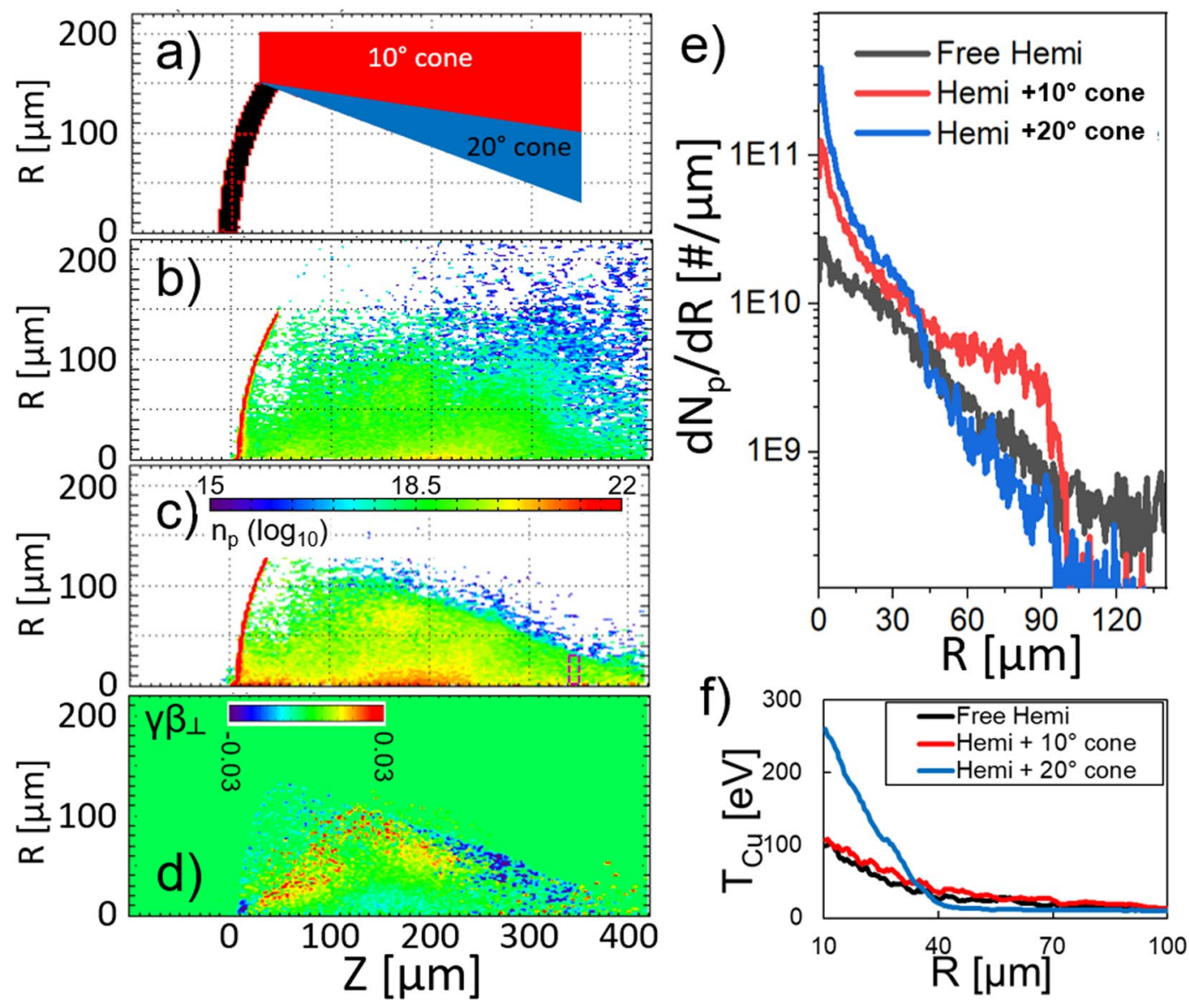

Figure 6. (a) simulated target geometry for the standard cone and 20 deg cone. (b) proton density for the free hemi at 22 ps. (c) proton density for the $20 \mathrm{deg}$ cone at $20 \mathrm{ps}$. (d) radial velocity of proton macroparticles for the $20 \mathrm{deg}$ cone at 16 ps. (e) the radial distribution of protons reaching the purple box shown in (c) through 33 ps. (f) $\mathrm{Cu}$ ion temperature radial profile for the same three cases.

\begin{abstract}
Methods
Experimental configuration. The experiment was conducted with orthogonal short pulse beams from OMEGA EP. The main beam (1250 J, $10 \mathrm{ps}, 35 \mu \mathrm{m} r_{80}$, where $r_{80}$ is the radius containing $80 \%$ of the spot energy) was incident on the target apex in line with the axis of symmetry of the spherical cap and the cone. A 3-dimensional representation of the target and important diagnostic lines of sight is shown in Fig. 2. The Spherical Crystal Imager $\left(\mathrm{SCI}^{37}\right)$ fielded a spherical quartz crystal from 63 deg above the equatorial plane, directly above the cone axis. The foil stacks were $600 \times 300 \mu \mathrm{m}$ and positioned either 300 or $450 \mu \mathrm{m}$ behind the planar portion of the hemi target. When present, the cone or wedge structure was directly glued to the hemi and the foil stack. The rear layer was a $10 \mu \mathrm{m}$ thick $\mathrm{Cu}$ foil; it was either uncoated or coated with a front layer of $13 \mu \mathrm{m} \mathrm{Al} \mathrm{or} 6 \mu \mathrm{m} \mathrm{Ag}$, the importance of which is beyond the scope of this paper. There were no measurable differences observed in the $K \alpha$ from targets with $\mathrm{Al}$ vs Ag. Their thicknesses were chosen to have the same stopping range for protons. The beam used for proton radiography $\left(850 \mathrm{~J}, 10 \mathrm{ps}, 20 \mu \mathrm{m} \mathrm{r}_{80}\right.$ ) was incident normally on a $1 \mathrm{~mm}$ square $10 \mu \mathrm{m}$ thick Au foil. A custom arrangement of radiochromic films was used with $100 \mu \mathrm{m}$ Al foil in front, 53 slices of film, and minimal filters between, to preserve a high degree of temporal discrimination.
\end{abstract}

Particle-in-cell simulations of particle generation, transport, and induced Cu K $\alpha$. Particle-in-cell code LSP was used to study kinetic aspects of the main target. The hemi + wedge target geometry was represented including a preplasma, $\mathrm{C}$ curved foil, $\mathrm{Al}$ trapezoidal sidewalls and rear foil. For simplicity, the rear foil was modeled as Al for the first simulation. The geometry is shown in Fig. 7(a). By using LSP's implicit push algorithm and the fluid description in the solid regions of the target and $\mathrm{Al}$ structures, the number of macroparticles/cell limits could be significantly relaxed comparing to typical PIC simulations. In these fluid regions, particles follow the same equation of motion of kinetic particles but a collision term using pressure and frictional forces is added. Meanwhile, the particles that are responsible for the TNSA effect (namely hot electrons and surface protons) are treated kinetically with high particle density, fully resolving the acceleration. In our simulations, 4 particles/cell for each species were laid out in the solid regions and 625 particles/cell filled the $1 \mu m$ thick hydrogen layer of the Hemi target. The minimum grid size was $0.4 \mu \mathrm{m}$ for non-uniform grids. Simulations outputted the snap shots of fields and particle densities every $0.5 \mathrm{ps}$. Data was accumulated on extraction planes every time step, $0.4 f \mathrm{~s}$. 


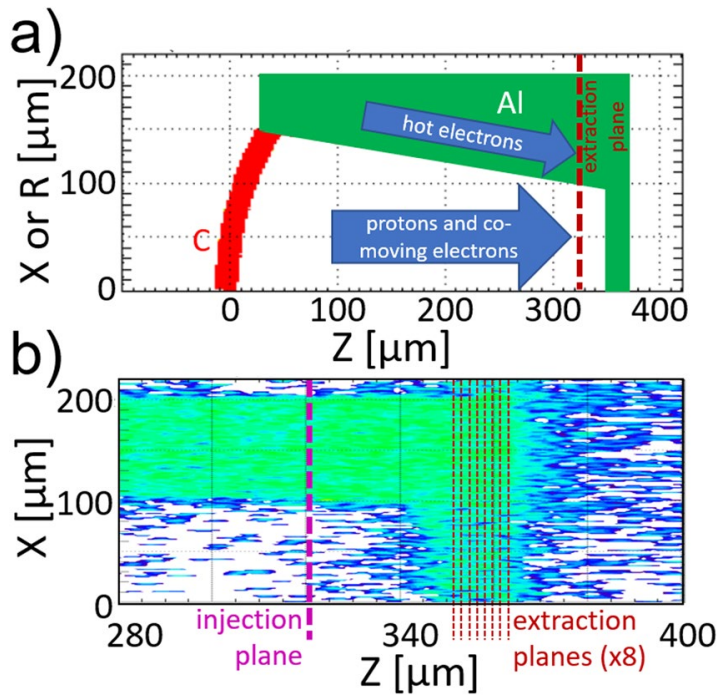

Figure 7. (a) The simulation geometry showing one half of the domain for a simulation in cartesian cordinates (X) or the whole domain for cylindrical coordinates (R). (b) Closeup of the $\mathrm{Cu}$ foil in a cartesian run, showing where hot electrons and protons are counted in the middle of the Cu region. The plotted quantity is electron density with the same color scale as Figs. 4(a) and 6(b,c).

To make the problem tractable with finite computational resources, the laser-target interaction process was bypassed- a population of electrons was injected to emulate the laser interaction. The electron source had total energy $30 \%$ of $1250 \mathrm{~J}=375 \mathrm{~J}$ in 20 ps total duration with 10 ps FW HM Gaussian history and $35 \mu m$ FW HM Gaussian spatial profile. The energy spectrum had slope temperature linearly increasing to $3 \mathrm{MeV}$ until the laser peak and a $30 \%$ Gaussian energy spread in a transverse direction. The electron source method has been applied previously in similar problems ${ }^{30}$ and benchmarked against full simulation of the laser ${ }^{31}$. The choice of an electron source with increasing slope temperature that exceeds that predicted by ponderomotive scaling for the laser intensity is a proven method for more accurately simulating proton acceleration from multi-picosecond pulses ${ }^{38}$. Longitudinal and lateral electric field maps were recorded periodically as were all species' densities, temperatures and velocities.

Electrons reached the rear foil primarily (in number) by traveling within the target structure and along the walls while protons drift through the gap. All particles, including protons, hot electrons, and co-moving electrons, that nearly reached the rear foil $(Z=325 \mu \mathrm{m})$ were recorded in the first simulation then input into a separate transport simulation at the recorded position in front of a $\mathrm{Cu}$ slab, preserving each particle's momentum, lateral position, and relative timing.

In the $\mathrm{Cu}$ transport simulations, the injection plane was set before the entrance of the transport layer $(\mathrm{Cu}$ slab) to enable multiple transport simulations without having to re-simulate the long time of flight of particles from the hemispherical target. The domain is shown in Fig. 7(b). The particles collected in the longer simulation were re-injected in the $\mathrm{Cu}$ transport simulation with the same temporal and spatial profiles as collected and at the same plane along the cone. The collection/injection plane was chosen in front of the $\mathrm{Cu}$ slab to avoid field effects at the slab boundary. A vacuum gap was included in the domain so that fields could evolve in time without numerical disturbance.

A series of extraction planes recorded the particles' momenta and positions every $2 \mu m$ into the slab. Particle stopping calculations in the slab applied Atzeni's approach for relativistic electrons ${ }^{39}$ and a bound plus free electronic stopping model for protons ${ }^{40}$. The PIXE cross-sections for electron- and proton-induced $\mathrm{Cu} K \alpha$ emis$\operatorname{sion}^{41,42}$ are applied with the simplifying assumption that the particle energies are constant over each $2 \mu m$ slice. The shape of the $\mathrm{Cu}$ k $\alpha$ emission profile can be plotted using the particle positions, and the cumulative profile that would be observed from the rear of the foil is then calculated by adding the slices, accounting for x-ray opacity.

Received: 3 December 2019; Accepted: 13 April 2020;

Published online: 10 June 2020

\section{References}

1. Hatchett, S. P. et al. Electron, photon, and ion beams from the relativistic interaction of petawatt laser pulses with solid targets. Physics of Plasmas 7, 2076 (2000).

2. Clark, E. L. et al. Energetic heavy-ion and proton generation from ultraintense laser-plasma interactions with solids. Phys. Rev. Lett. 85, $1654(2000)$.

3. Wilks, S. C. et al. Energetic proton generation in ultra-intense laser--solid interactions. Physics of Plasmas 8, 542 (2001).

4. Patel, P. K. et al. Isochoric heating of solid-density matter with an ultrafast proton beam. Phys. Rev. Lett. 91, 125004 (2003).

5. Snavely, R. A. et al. Laser generated proton beam focusing and high temperature isochoric heating of solid matter. Physics of Plasmas 14, 092703 (2007).

6. Smith, R. F. et al. Ramp compression of diamond to five terapascals. Nature https://doi.org/10.1038/nature13526. (2014), 
7. Roth, M. et al. Energetic ions generated by laser pulses: A detailed study on target properties. Phys. Rev. ST Accel. Beams 5, 061301 (2002).

8. Borghesi, M. et al. Electric field detection in laser-plasma interaction experiments via the proton imaging technique. Physics of Plasmas 9, 2214 (2002).

9. Romagnani, L. et al. Dynamics of electric fields driving the laser acceleration of multi-mev protons. Phys. Rev. Lett. 95, 195001 (2005).

10. Fuchs, J. et al. Comparative spectra and efficiencies of ions laser-accelerated forward from the front and rear surfaces of thin solid foils. Physics of Plasmas 14, 053105 (2007).

11. Quinn, K. et al. Laser-driven ultrafast field propagation on solid surfaces. Phys. Rev. Lett. 102, 194801 (2009).

12. Mackinnon, A. J. et al. Proton radiography of a laser-driven implosion. Phys. Rev. Lett. 97, 045001 (2006).

13. Higginson, D. P. et al. Production of neutrons up to $18 \mathrm{mev}$ in high-intensity, short-pulse laser matter interactions. Physics of Plasmas (1994-present) 18, 100703 (2011).

14. Roth, M. et al. Bright laser-driven neutron source based on the relativistic transparency of solids. Phys. Rev. Lett. 110, 044802 (2013).

15. Roth, M. et al. Fast ignition by intense laser-accelerated proton beams. Phys. Rev. Lett. 86, 436 (2001).

16. Key, M. H. et al. Proton fast ignition. Fusion Science and Technology 49, 440, https://doi.org/10.13182/FST06-A1160 (2006).

17. Roth, M. et al. Proton acceleration experiments and warm dense matter research using high power lasers. Plasma Physics and Controlled Fusion 51, 124039 (2009).

18. Cowan, T. E. et al. Ultralow emittance, multi-mev proton beams from a laser virtual-cathode plasma accelerator. Phys. Rev. Lett. 92, 204801 (2004).

19. Zou, D. B. et al. Enhanced target normal sheath acceleration based on the laser relativistic self-focusing. Physics of Plasmas (1994-present) 21, 063103 (2014).

20. Yin, L. et al. Three-dimensional dynamics of breakout afterburner ion acceleration using high-contrast short-pulse laser and nanoscale targets. Phys. Rev. Lett. 107, 045003 (2011).

21. Hegelich, B. et al. 160 mev laser-accelerated protons from ch2 nano-targets for proton cancer therapy. arXiv preprint arXiv:1310.8650 (2013).

22. Green, J. S. et al. High efficiency proton beam generation through target thickness control in femtosecond laser-plasma interactions. Applied Physics Letters 104, 214101 (2014).

23. Wagner, F. et al. Maximum proton energy above $85 \mathrm{mev}$ from the relativistic interaction of laser pulses with micrometer thick $\mathrm{ch}_{2}$ targets. Phys. Rev. Lett. 116, 205002 (2016).

24. Higginson, A. et al. Near-100 MeV protons via a laser-driven transparency-enhanced hybrid acceleration scheme. Nature Communications 9, 724 (2018)

25. Bartal, T. et al. Proton Focusing Characteristics Relevant to Fast Ignition. IEEE Transactions on Plasma Science 39, 2818 (2011).

26. Patel, P. K. et al. Integrated laser-target interaction experiments on the ral petawatt laser. Plasma Physics and Controlled Fusion 47, B833 (2005).

27. Bellei, C. et al. Electron and ion dynamics during the expansion of a laser-heated plasma under vacuum. Physics of Plasmas (1994-present) 19, 033109 (2012).

28. Bartal, T. et al. Focusing of short-pulse high-intensity laser-accelerated proton beams. Nature Physics 8, 139 (2012).

29. Offermann, D. T. et al. Characterization and focusing of light ion beams generated by ultra-intensely irradiated thin foils at the kilojoule scale. Physics of Plasmas 18, 056713 (2011).

30. Foord, M. E. et al. Proton trajectories and electric fields in a laser-accelerated focused proton beam. Physics of Plasmas (1994-present) 19, 056702 (2012)

31. Qiao, B. et al. Dynamics of high-energy proton beam acceleration and focusing from hemisphere-cone targets by high-intensity lasers. Phys. Rev. E 87, 013108 (2013).

32. Kar, S. et al. Guided post-acceleration of laser-driven ions by a miniature modular structure. Nature Communications 7, 10792 (2016).

33. Chaiwai, C., Yu, L. \& Tippawan, U. Pixe-measurement of ka x-ray production cross sections for 1-mev c+-ions in thick samples of si, fe, cu and zn. Nuclear Instruments and Methods in Physics Research Section B: Beam Interactions with Materials and Atoms 450, 279, the 23rd International Conference on Ion Beam Analysis (2019).

34. Prieto, J. E., Zucchiatti, A., Galn, P. \& Prieto, P. Cross sections of $\mathrm{x}$-ray production induced by $\mathrm{c}$ and si ions with energies up to $1 \mathrm{mev} / \mathrm{u}$ on ti, fe, $\mathrm{zn}, \mathrm{nb}$, ru and ta. Nuclear Instruments and Methods in Physics Research Section B: Beam Interactions with Materials and Atoms 406, 167, proceedings of the 12th European Conference on Accelerators in Applied Research and Technology (ECAART12) (2017)

35. Welch, D. R., Rose, D. V., Cuneo, M. E., Campbell, R. B. \& Mehlhorn, T. A. Integrated simulation of the generation and transport of proton beams from laser-target interaction. Physics of Plasmas (1994-present) 13, 063105 (2006).

36. Kim, J. et al. Varying stopping and self-focusing of intense proton beams as they heat solid density matter. Physics of Plasmas 23, 043104, https://doi.org/10.1063/1.4945617 (2016).

37. Koch, J. A. et al. 4.5- and 8-kev emission and absorption $\mathrm{x}$-ray imaging using spherically bent quartz 203 and 211 crystals (invited). Review of Scientific Instruments 74, 2130, https://doi.org/10.1063/1.1537448 (2003).

38. Kim, J. et al. Computational modeling of proton acceleration with multi-picosecond and high energy, kilojoule, lasers. Physics of Plasmas 25, 083109, https://doi.org/10.1063/1.5040410 (2018).

39. Atzeni, S., Schiavi, A. \& Davies, J. R. Stopping and scattering of relativistic electron beams in dense plasmas and requirements for fast ignition. Plasma Physics and Controlled Fusion 51, 015016 (2009).

40. Kim, J. et al. Self-consistent simulation of transport and energy deposition of intense laser-accelerated proton beams in solid-density matter. Phys. Rev. Lett. 115, 054801 (2015).

41. Hombourger, C. An empirical expression for K-shell ionization cross section by electron impact. Journal of Physics B Atomic Molecular Physics 31, 3693 (1998).

42. Paul, H. \& Sacher, J. Fitted empirical reference cross sections for k-shell ionization by protons. Atomic Data and Nuclear Data Tables 42, 105 (1989).

\section{Acknowledgements}

This material is based upon work supported by the U.S. Department of Energy National Nuclear Security Agency under the National Laser User Facility (NLUF) program with Award Number DE-NA0002034 and the High Energy Density Laboratory Plasmas program with Award Number DE-NA0003876. The support of DOE does not constitute an endorsement by DOE of the views expressed in this article. The authors would like to acknowledge excellent support provided by the OMEGA EP Laser Facility staff including D. Canning and D. Mastrosimone and General Atomics target fabrication by Michelle Evans. We thank B. Qiao for serving as NLUF PI while at UCSD. S.N.C is supported by Extreme Light Infrastructure Nuclear Physics (ELI-NP) Phase II, a project co-financed by the Romanian Government and European Union through the European Regional Development Fund. JF is supported by Grant ANR-17-CE30- 0026-Pinnacle from Agence Nationale de la Recherche and by the European 
Research Council (ERC) under the European Union's Horizon 2020 research and innovation program (Grant Agreement No. 787539).

\section{Author contributions}

C.M. designed the experiment with help from M.S.W., P.M.N., S.N.C., J.F., F.N.B., H.S.M. and P.K.P. The new targets were manufactured by P.F. based on ideas by M.E.F., R.B.S. and F.N.B. Data analyses were performed by C.M. and J.K. Simulations were performed by J.K. and D.M. C.M. and J.K. led the writing of the manuscript.

\section{Competing interests}

The authors declare no competing interests.

\section{Additional information}

Supplementary information is available for this paper at https://doi.org/10.1038/s41598-020-65554-4.

Correspondence and requests for materials should be addressed to C.M.

Reprints and permissions information is available at www.nature.com/reprints.

Publisher's note Springer Nature remains neutral with regard to jurisdictional claims in published maps and institutional affiliations.

(1) Open Access This article is licensed under a Creative Commons Attribution 4.0 International License, which permits use, sharing, adaptation, distribution and reproduction in any medium or format, as long as you give appropriate credit to the original author(s) and the source, provide a link to the Creative Commons license, and indicate if changes were made. The images or other third party material in this article are included in the article's Creative Commons license, unless indicated otherwise in a credit line to the material. If material is not included in the article's Creative Commons license and your intended use is not permitted by statutory regulation or exceeds the permitted use, you will need to obtain permission directly from the copyright holder. To view a copy of this license, visit http://creativecommons.org/licenses/by/4.0/.

(C) The Author(s) 2020 